\title{
TITLE:
}

\section{Light-Emitting Waveguide-Plasmon Polaritons}

\section{$\operatorname{AUTHOR}(\mathrm{S})$ :}

Rodriguez, S. R. K.; Murai, S.; Verschuuren, M. A.; Rivas, J. Gomez

\section{CITATION:}

Rodriguez, S. R. K. ...[et al]. Light-Emitting Waveguide-Plasmon Polaritons. Physical Review Letters 2012, 109(16): 166803.

ISSUE DATE:

2012-10-17

URL:

http://hdl.handle.net/2433/188015

RIGHT:

(C)2012 American Physical Society 


\title{
Light-Emitting Waveguide-Plasmon Polaritons
}

\author{
S. R. K. Rodriguez, ${ }^{1, *}$ S. Murai, ${ }^{1,2, \dagger}$ M. A. Verschuuren, ${ }^{3}$ and J. Gómez Rivas ${ }^{1,4}$ \\ ${ }^{1}$ Center for Nanophotonics, FOM Institute AMOLF, clo Philips Research Laboratories, \\ High Tech Campus 4, 5656 AE Eindhoven, The Netherlands \\ ${ }^{2}$ Department of Material Chemistry, Graduate School of Engineering, Kyoto University, Katsura, Nishikyo-ku, Kyoto 615-8510, Japan \\ ${ }^{3}$ Philips Research Laboratories, High Tech Campus 4, 5656 AE Eindhoven, The Netherlands \\ ${ }^{4}$ COBRA Research Institute, Eindhoven University of Technology, P.O. Box 513, 5600 MB Eindhoven, The Netherlands
}

(Received 28 June 2012; published 17 October 2012)

\begin{abstract}
We demonstrate the generation of light in an optical waveguide strongly coupled to a periodic array of metallic nanoantennas. This coupling gives rise to hybrid waveguide-plasmon polaritons (WPPs), which undergo a transmutation from plasmon to waveguide mode and vice versa as the eigenfrequency detuning of the bare states transits through zero. Near zero detuning, the structure is nearly transparent in the far-field but sustains strong local field enhancements inside the waveguide. Consequently, light-emitting WPPs are strongly enhanced at energies and in-plane momenta for which WPPs minimize light extinction. We elucidate the unusual properties of these polaritons through a classical model of coupled harmonic oscillators.
\end{abstract}

DOI: 10.1103/PhysRevLett.109.166803

PACS numbers: 73.20.Mf, 05.45.Xt, 42.82.Et, 78.67. $-\mathrm{n}$

The field of plasmonic metamaterials has inherited an invaluable legacy from atomic physics for the interpretation of nontrivial spectral line shapes. Seminal examples are Fano's theory on asymmetric resonance line shapes [1], and Electromagnetically Induced Transparency (EIT) [2]. Fano described the quantum interference between a continuum of states and a discrete state whose energy lies within the continuum. His theory has found broad applicability to classical systems also [3,4], where similar line shapes arise from the interaction of spectrally broad and narrow resonances. The connected phenomenon of EIT, wherein destructive quantum interference between different excitation pathways renders a spectrally narrow transparency window within an absorbtion band of an atomic medium [5,6], has also been a major inspiration for the metamaterials community [7-12]. The abrupt changes in frequency dispersion associated with EIT enable light to be slowed [13], or even halted [14]. Despite the fundamental distinction in the nature of quantum versus classical waves, i.e., probability amplitudes rather than classical field amplitudes interfere, Fano, EIT, and even tunneling-like behavior $[15,16]$ manifest for both due to the ubiquity of wave phenomena.

In this Letter, we demonstrate the generation of light in a strongly coupled classical system displaying Fano and EITlike spectral line shapes. We investigate the coupling of localized surface plasmon polaritons (LSPPs), i.e., collective oscillations of conduction electrons in metallic nanostructures, to a guided mode in a light-emitting slab. LSPPs enable the conversion of free space radiation into localized energy and vice versa, empowering metallic nanostructures as antennas for light $[17,18]$. When a waveguide is placed near nanoantennas, the strong coupling of LSPPs to guided modes leads to the formation of a new quasiparticle in light extinction, known already as a waveguide-plasmon polariton (WPP) [19]. We herein report on a novel class of light-emitting WPPs which display an extraordinarily enhanced emission at frequencies of strong dispersion and nearly perfect far-field transparency. We support our experimental findings with numerical simulations and a coupled oscillator model, explaining how light emission can be resonantly enhanced in nearly transparent optical media. Emission enhancements at energies and in-plane momenta of far-field transparency hold great promise for plasmonic based solid-state light-emitting devices with negligible absorption losses. By reciprocity, a sensor can be cloaked such that its local sensitivity is enhanced while remaining invisible to distant observers [20,21].

Figure 1(a) illustrates the structure we investigate. As a light-emitting slab waveguide, a $230 \mathrm{~nm}$ layer of yttrium aluminum garnet doped with $\mathrm{Ce}^{3+}$ ions (YAG:Ce) was fabricated by a sol-gel method onto a fused silica substrate as described in Ref. [22]. The $\sim 0.4 \mathrm{eV}$ full width at half maximum (FWHM) emission of YAG:Ce allows us to study light-emitting WPPs in a wide spectral range. A $20 \mathrm{~nm}$ layer of $\mathrm{Si}_{3} \mathrm{~N}_{4}$ was deposited on top of the slab for a twofold purpose: (i) to planarize the surface, and (ii) to avoid emission quenching of $\mathrm{Ce}^{3+}$ ions in proximity to the metal $[23,24]$. An Ag nanoantenna array with a size of $2 \times 2 \mathrm{~mm}^{2}$ was fabricated by substrate conformal imprint lithography [25] onto the $\mathrm{Si}_{3} \mathrm{~N}_{4}$ layer. Figure 1(b) shows a scanning electron microscope image of the array. The dimensions of the nanoantennas are $90 \times 70 \times 20 \mathrm{~nm}^{3}$, and the lattice constants are $a_{x}=$ $300 \mathrm{~nm}$ and $a_{y}=200 \mathrm{~nm}$. The array was covered by a conformal layer of $\mathrm{Si}_{3} \mathrm{~N}_{4}$ with a thickness of $20 \mathrm{~nm}$ to protect the $\mathrm{Ag}$ nanoantennas from oxidation. We will show later (Fig. 4) that, although $\mathrm{Si}_{3} \mathrm{~N}_{4}(n \approx 2.0)$ has a higher refractive index than YAG:Ce $(n \approx 1.7$, as determined from ellipsometry) in the part of the visible spectrum 


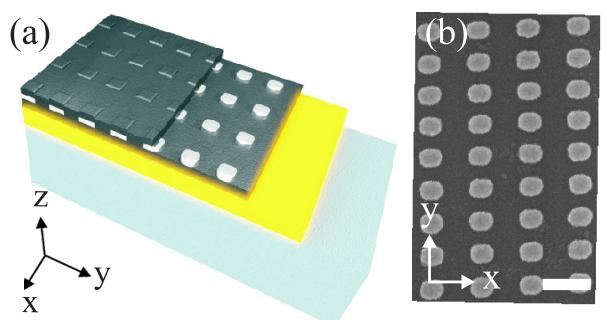

FIG. 1 (color online). (a) 3D view of the light-emitting waveguide structure coupled to a silver nanoantenna array. The layers extend up to different positions along the $y$ axis for illustration purposes only. From bottom to top, the layers are, $1 \mathrm{~mm}$ of $\mathrm{SiO}_{2}$, $230 \mathrm{~nm}$ of YAG:Ce, and $20 \mathrm{~nm}$ silver nanoantennas surrounded by $20 \mathrm{~nm}$ of $\mathrm{Si}_{3} \mathrm{~N}_{4}$ on top and bottom. The light field emitted from the YAG:Ce couples localized surface plasmon polaritons in the silver nanoantennas to guided photons in the YAG:Ce slab waveguide. (b) SEM image of the silver nanoantenna array. The scale bar denotes $300 \mathrm{~nm}$.

where we work, the thinness of the $\mathrm{Si}_{3} \mathrm{~N}_{4}$ layer and the presence of the metallic antennas enables the excitation of a guided mode in the YAG:Ce layer.

We performed variable angle, polarization-resolved, extinction spectroscopy. The white light from a halogen lamp was collimated and linearly polarized parallel to the short axis of the nanoantennas ( $y$ axis in Fig. 1). The sample was rotated around the $y$ axis, resulting in $s$-polarized incidence. The zeroth-order transmittance $T_{0}$ was collected by a fiber-coupled spectrophotometer. The extinction, defined as $1-T_{0}$, is shown in Fig. 2(a) as a function of the incident photon energy and the wave vector component parallel to the long axis of the antennas, $\mathbf{k}_{\|}=k_{0} \sin \left(\theta_{\text {in }}\right) \hat{x}$, with $k_{0}$ the free space wave vector and $\theta_{\text {in }}$ the angle of incidence. Computer controlled rotation stages with an
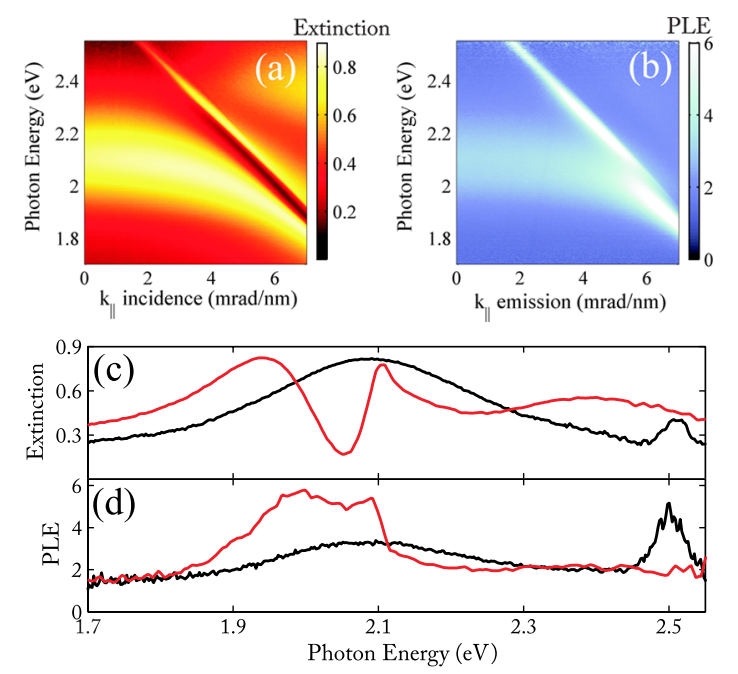

FIG. 2 (color online). (a) Extinction and (b) PhotoLuminescence Enhancement (PLE) of the emission from the YAG:Ce layer coupled to the antennas, normalized to the layer without antennas. (c) and (d) are cuts of (a) and (b), respectively, at $k_{\|}=2.1 \mathrm{mrad} / \mathrm{nm}$ (black lines) and $k_{\|}=5.5 \mathrm{mrad} / \mathrm{nm}$ [red (grey) lines]. angular resolution $<0.2^{\circ}\left(k_{\|} \approx 35 \mu \mathrm{rad} / \mathrm{nm}\right.$ at $\left.2 \mathrm{eV}\right)$ were used for the measurements. We shall refer to the magnitude of $\mathbf{k}_{\|}$as $k_{\|}$.

The feature near $2.1 \mathrm{eV}$ at normal incidence in Fig. 2(a) is associated with the dipolar LSPP along the short axis of the antennas. Its flat angular dispersion and broad linewidth are characteristic of localized resonances. The small size of the particle along the polarization axis $(70 \mathrm{~nm})$ determines the primarily dipolar response. The narrow feature on the high energy side corresponds to the fundamental $T E$ guided mode in the YAG:Ce slab. Its dispersion has been folded onto the first Brillouin zone by the periodic nanoantenna array. As $k_{\|}$increases, the LSPP acquires an angularly dispersive character and a narrower linewidth, whereas the opposite occurs for the guided mode. Thus, their properties are interchanged.

To obtain the light emission dispersion diagram, the sample was photoexcited by a continuous wave laser with an energy of $2.8 \mathrm{eV}$, FWHM $=30 \mathrm{meV}$, a power far below saturation, impinging at an angle of $10^{\circ}$. The photoluminescence passed through an analyzer, and was collected as a function of the angle $\theta_{\mathrm{em}}$ subtended by the detector and the normal to the sample. The detector was rotated around the $y$ axis, collecting $s$-polarized light with $\mathbf{k}_{\|}=k_{0} \sin \left(\theta_{\mathrm{em}}\right) \hat{x}$. The angular resolution was the same as in the extinction measurements. The sample was excited nonresonantly with regards to the plasmonic structure, as will be shown next.

Figure 2(b) shows the measured photoluminescence enhnancement (PLE), defined as $I_{\text {in }} / I_{\text {out }}$ with $I_{\text {in }}$ and $I_{\text {out }}$ the emission from the YAG:Ce slab in and out of the presence of the nanoantenna array, respectively. For small $k_{\|}$, where the LSPP and guided mode resonances are largely detuned, the PLE displays features qualitatively resembling those in extinction. Near zero detuning, occurring for $k_{\|} \approx 5.5 \mathrm{mrad} / \mathrm{nm}$, a remarkable contrast between extinction and PLE takes place. We illustrate examples of large detuning $k_{\|}=2.1 \mathrm{mrad} / \mathrm{nm}$ (black line) and near zero detuning $k_{\|}=5.5 \mathrm{mrad} / \mathrm{nm}$ [red (grey) line], for light extinction in Fig. 2(c) and PLE in Fig. 2(d). For large detuning, the broad and strong extinction feature associated with the LSPP leads to a modest PLE with a similar line shape. The narrow and weak feature with a Fano-like line shape associated with the guided mode leads to a larger PLE. This contrasting behavior has its origin in the spatial field distributions associated with the LSPP and guided mode, which we illustrate later by means of simulations. Near zero detuning, it is not possible to discriminate between the LSPP and guided mode. In this case, the extinction displays an EIT-like line shape with a nearly perfect transparency window. Remarkably, the emission from the YAG:Ce waveguide is largely enhanced in this far-field transparent and strongly dispersive spectral region, resulting in a strong and spectrally broad light-emitting WPP. Notice that far from any resonance the PLE tends to 1 . This indicates that pump enhancements, i.e., resonant processes 
at the excitation energy, are negligible. By matching the momentum of the pump photons to the momentum of the waveguide photons with the assistance of the grating, the magnitude of the PLE could be further boosted while preserving the dispersive properties of the light-emitting WPPs. Alternatively, the pump photons may be evanescently coupled into the YAG:Ce waveguide as recently shown in Ref. [22]. Therein, a 30-fold pump enhancement was reported with negligible enhancement at the emission energies.

We performed finite element method simulations (COMSOL) under plane wave illumination to illustrate the field distributions associated with the coupled optical modes. For ease of computation, we chose a $2 \mathrm{D}$ system with the same periodicity as the experimental structure along the $x$ axis (the direction of the in-plane momentum). The width and height of the simulated metallic wire were chosen to be $70 \mathrm{~nm}$ and $20 \mathrm{~nm}$, respectively. These dimensions determine the spectral features of the LSPP. For $T M$ polarization, the coupling between the LSPPs along the width of the metallic wire and the $T M$ guided mode gives rise to the same physics in light extinction observed in our 3D system for $T E$ polarization [19]. The optical data for $\mathrm{Ag}$ was taken from Ref. [26], for YAG:Ce it was taken from ellipsometry measurements not shown here, and we set a constant refractive index of $n=2.0$ for $\mathrm{Si}_{3} \mathrm{~N}_{4}$ and $n=1.43$ for $\mathrm{SiO}_{2}$. Floquet boundary conditions were used for the sides of the unit cell, and perfectly matched layers on the top and bottom.

Figure 3(a) shows the extinction obtained from the simulations. The white dashed line indicates the dispersion relation of the fundamental $T M$ guided mode in a threelayer structure, calculated as outlined in Ref. [27]. The structure consists of $\mathrm{SiO}_{2}$ with $n=1.43$, an effective YAGCe $+\mathrm{Si}_{3} \mathrm{~N}_{4}$ slab assuming their average refractive index weighted by the corresponding fractional area, and air with $n=1$. The good agreement between the white line and the transparency band in Fig. 3(a), arising at the eigenvalue of the (bare) guided mode, justifies the assumption for determining the refractive index of the middle layer. Figure 3(b) shows the simulated electric field intensity enhancement in the YAG:Ce slab, defined as $|E|^{2} /\left|E_{0}\right|^{2}$ with $E$ and $E_{0}$ the total and incident electric field, respectively, both spatially averaged over the interior of the YAG: Ce slab. In Figs. 3(c) and 3(d) we make cuts of the extinction and $|E|^{2} /\left|E_{0}\right|^{2}$, respectively, at a large detuning of $k_{\|}=$ $2.1 \mathrm{mrad} / \mathrm{nm}$ (black lines) and near zero detuning of $k_{\|}=$ $5.5 \mathrm{mrad} / \mathrm{nm}$ [red (grey) lines]. At a large detuning we observe that $|E|^{2} /\left|E_{0}\right|^{2}$ is significantly greater at the guided mode energy $(2.4 \mathrm{eV})$ than at the LSPP energy $(2.05 \mathrm{eV})$. The large electromagnetic enhancements occurring in the locality of the emitters explain the greater emission enhancement measured for the guided mode at large detunings. Near zero detuning, $|E|^{2} /\left|E_{0}\right|^{2}$ displays a maximum at energies within the far-field transparency band. This counterintuitive behavior arises from the interference nature of the extinction, which allows for a partial cancelation of the
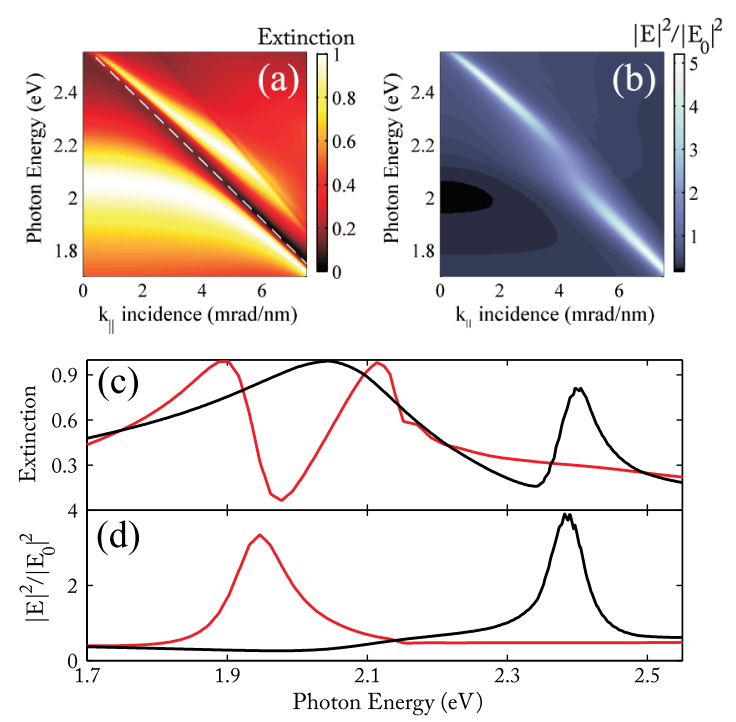

FIG. 3 (color online). Finite element method simulations of (a) the extinction of an array of metallic nanowires with $70 \mathrm{~nm}$ width and $300 \mathrm{~nm}$ periodicity, and (b) the corresponding average field intensity enhancement in the YAG:Ce slab. (c) and (d) are cuts of (a) and (b), respectively, at $k_{\|}=2.1 \mathrm{mrad} / \mathrm{nm}$ (black lines) and $k_{\|}=5.5 \mathrm{mrad} / \mathrm{nm}$ [red (grey) lines].

far-field response of a system sustaining intense local fields. This property of extinction is at the heart of recent theoretical proposals for cloaking a sensor [20,21]. It transpires that near zero detuning the LSPP-guided-mode coupling induces a transparency band through far-field interference, yet the emission from the $\mathrm{Ce}^{3+}$ ions is enhanced due to the local field enhancements shown in Fig. 3.

We present in Fig. 4 the total electric field normalized to the incident field at the same values of large detuning [panels (a)-(c)] and near zero detuning [panels (d)-(e)] inspected in Figs. 3(c) and 3(d). Figures 4(a) and 4(d) correspond to the high energy extinction peak, Figs. 4(b) and 4(e) correspond to the extinction dip, and Figs. 4(c) and 4(f) correspond to the low energy extinction peak. At large detuning the field is highly concentrated in the YAG:Ce layer for (a) - the guided mode-whereas it is more concentrated near the metallic structure for (c) - the LSPP. Figure 4(a) shows that the guided mode resides primarily in the YAG:Ce waveguide, with only a small fraction of the total field enhancement in the $\mathrm{Si}_{3} \mathrm{~N}_{4}$ layer. Near zero detuning, the field distributions in (d) and (f) are very similar, since the high and low energy extinction peaks correspond to hybrid states with approximately equal weights. Figure 4(e) shows strong local field enhancements in the YAG:Ce layer at an energy and wave vector for which the structure is nearly transparent. Comparing Figs. 4(e) to 4(b) reveals the critical role of the eigenmode detuning in the average field enhancement inside the waveguide at the energy of the extinction minimum. Whereas only a small enhancement occurs for large detunings, a much larger enhancement occurs near zero detuning, resulting in a more intense emission at the latter condition. 


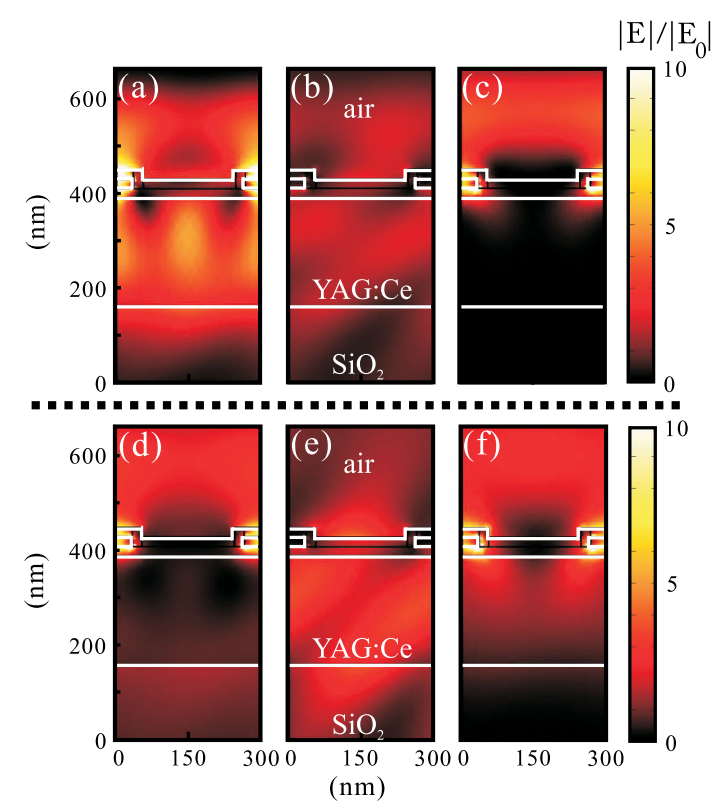

FIG. 4 (color online). Field enhancement for large waveguideplasmon detuning (a)-(c), i.e., at $k_{\|}=2.1 \mathrm{mrad} / \mathrm{nm}$, and near zero detuning (d)-(f), i.e., at $k_{\|}=5.5 \mathrm{mrad} / \mathrm{nm}$. (a) and (d) correspond to the high energy extinction peak, (b) and (e) correspond to the extinction dip, and (c) and (f) correspond to the low energy extinction peak, all referring to the spectra in Fig. 3(c). The metallic antennas, surrounded by $20 \mathrm{~nm}$ of $\mathrm{Si}_{3} \mathrm{~N}_{4}$, are located at the interface between the YAG:Ce layer and air.

We elucidate the physics involved in the LSPP-guidedmode coupling through a model of coupled harmonic oscillators. Classical analogs to EIT [28], Fano resonances [29], and strong coupling [16], have been presented with similar models. The model consists of two harmonic oscillators, one with eigenfrequency $\omega_{0}$, and the second with an eigenfrequency that is a function of the detuning $\delta$ from the eigenfrequency of the first oscillator, i.e., $\omega_{0}-\delta$. Assuming time-harmonic solutions, the coupled oscillator Hamiltonian is,

$$
\left(\begin{array}{cc}
\omega_{0}^{2}-\omega^{2}-i \gamma_{1} \omega & -\Omega_{12}^{2} \\
-\Omega_{12}^{2} & \left(\omega_{0}-\delta\right)^{2}-\omega^{2}-i \gamma_{2} \omega,
\end{array}\right),
$$

where $\gamma_{1,2}$ are the damping frequencies and $\Omega_{12}$ is the coupling frequency. In analogy to the extinction measurements, we drive the first oscillator directly by a harmonic force $F=F_{0} e^{-i \omega_{d} t}$, and calculate the real part of its dissipated power given by $P_{1}=\frac{1}{2} \operatorname{Re}\left\{F^{*} \dot{x}_{1}\right\}$, with $\dot{x}_{1}$ the velocity. We consider that only the first oscillator is driven directly by the harmonic force $F$ because only the LSPP is driven directly by the incident electromagnetic field. Since the dispersion relation of the guided mode lies below the light line, it can only be excited through the antennas. Finally, we integrate the dissipated power over one period of oscillation and scan the driving frequency $\omega_{d}$ to obtain the dissipated power spectrum, which we plot as a function of $\delta$ in Fig. 5(a). We set the eigenfrequency of the first oscillator to $\omega_{0}=2.12 \mathrm{rad} / \mathrm{s}$, the damping frequencies to $\gamma_{1}=0.4 \mathrm{rad} / \mathrm{s}, \gamma_{2}=0.02 \mathrm{rad} / \mathrm{s}$, the coupling frequency to $\Omega_{12}=0.58 \mathrm{rad} / \mathrm{s}$, and the force per unit mass to $F_{0}=$ $0.84 \mathrm{~m} / \mathrm{s}^{2}$. For the first oscillator we have $Q_{1}=\omega_{0} / \gamma_{1}=5$, which is typical for damped LSPPs [30]. The lower damping of the second oscillator reflects the lower losses of the waveguide, wherein absorption is nearly negligible and outof-plane scattering losses damp the mode relatively weakly. The coupling frequency and driving force amplitude were chosen to match the experiments.

The eigenfrequencies of the uncoupled and coupled oscillators are shown as solid and dashed lines in Fig. 5(a), respectively. The eigenfrequencies of the coupled system,

$$
\omega_{ \pm}^{2}=\frac{1}{2}\left[\omega_{0}^{2}+\left(\omega_{0}-\delta\right)^{2} \pm \sqrt{\left[\omega_{0}^{2}+\left(\omega_{0}-\delta\right)^{2}\right]^{2}-4\left[\omega_{0}^{2}\left(\omega_{0}-\delta\right)^{2}-\Omega_{12}^{4}\right]}\right]
$$

are obtained from Eq. (1) by letting $\gamma_{1,2}=0$ and solving for the characteristic equation. $\omega_{ \pm}$display an avoided crossing, in analogy to the quantum mechanical dressed states [31]. Their energy splitting at zero detuning is the classical counterpart of the Rabi splitting. Notice that for large detunings, $\omega_{ \pm}$asymptotically approach one of the eigenfrequencies of the uncoupled oscillators, i.e., the bare states. As $\omega_{ \pm}$each transit through $\delta=0$, they interchange their resemblance to one or the other of the uncoupled states. At $\delta=0, \omega_{ \pm}$cannot be associated with either one of the uncoupled oscillators, since the system resides in a hybrid (dressed) state of equal weights.

To model the local field intensity enhancement $|E|^{2} /\left|E_{0}\right|^{2}$ in the waveguide, we calculate the amplitude squared response of the associated oscillator, i.e., $\left|x_{2}\right|^{2}$. It was recently shown that the amplitude resonance of a single harmonic oscillator may be associated with the near-field resonance of a metallic nanoparticle [32]. Moreover, the shift in the amplitude response with respect to the dissipated power accounts for the shift of the near field with respect to the far-field [32]. We herein extend this principle to a coupled system, and show in Fig. 5(b) the dependence of this near-field analogous quantity on the detuning parameter. In Figs. 5(c) and 5(d) we make cuts of both Figs. 5(a) and 5(b) at a large detuning of $\delta=$ $-0.36 \mathrm{rad} / \mathrm{s}$ (black line) and at zero detuning [red (grey) line]. In its simplicity, the coupled oscillator model conveys the core of the physics involved. When the eigenfrequencies of the uncoupled oscillators are largely detuned, the dissipated power spectrum exhibits a Fano-like line shape near the eigenfrequency of the second oscillator. The enhanced amplitude response of the second oscillator near this feature explains the large emission enhancements observed near the guided mode resonance for large 

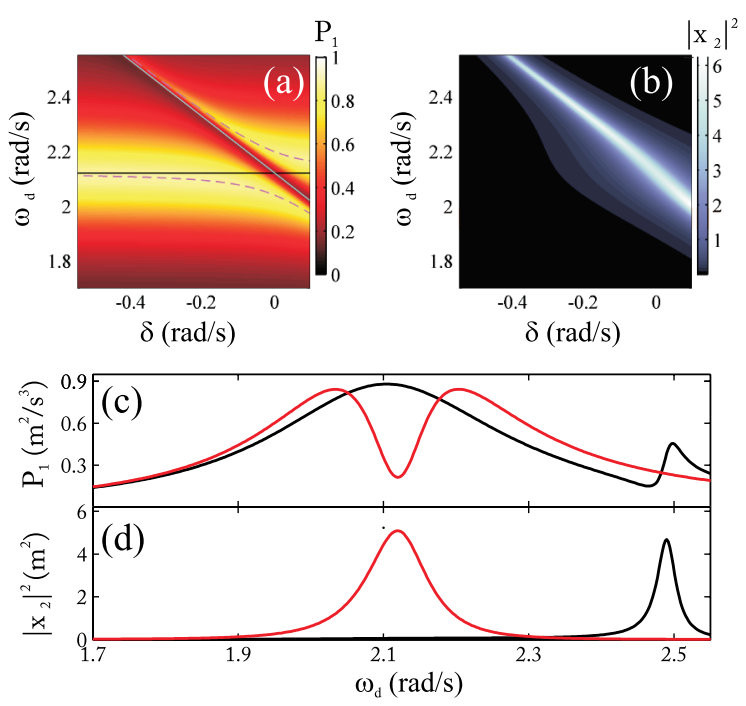

FIG. 5 (color online). (a) Power dissipated by the first oscillator, and (b) amplitude squared response of the second oscillator, both calculated from Eq. (1). (c) and (d) are cuts of (a) and (b), respectively, at $\delta=-0.36 \mathrm{rad} / \mathrm{s}$ (black lines) and $\delta=0$ [red (grey) lines]. In (a) the black solid line represents the eigenfrequency of the first uncoupled oscillator, the solid cyan line corresponds to the second uncoupled oscillator, and the magenta dashed lines are $\omega_{ \pm}$calculated from Eq. (2).

detunings. Moreover, the model shows that at zero detuning the coupled system displays a minimum in the dissipated power by one of its constituents (the first oscillator representing the driven electrons in the metal), while the amplitude response of the other constituent (the second oscillator representing the guided mode) is enhanced.

In conclusion, we have demonstrated the strong coupling of LSPPs to a guided mode in a light emitting slab. The properties of the emerging light-emitting WPPs were shown to be strongly dependent on the relative detuning between the two eigenmodes involved. Near zero detuning, light emission in the waveguide is resonantly enhanced in a strongly dispersive spectral region which is nearly transparent to the far-field. Our results open a new avenue for investigating EIT-related phenomena, i.e., slow light, strong dispersion, and coupling-induced transparencies, in the context of light emission.

This work was supported by the Netherlands Foundation for Fundamental Research on Matter (FOM) and the Netherlands Organisation for Scientific Research (NWO), and is part of an industrial partnership program between Philips and FOM. We thank G. Pirruccio for assistance in the simulations. S. Murai acknowledges financial support from Young Researchers Overseas Visits Program for Vitalizing Brain Circulation of JSPS, Japan.

\footnotetext{
*s.rodriguez@amolf.nl

†murai@dipole7.kuic.kyoto-u.ac.jp

[1] U. Fano, Phys. Rev. 124, 1866 (1961).
}

[2] K.-J. Boller, A. Imamoglu, and S. E. Harris, Phys. Rev. Lett. 66, 2593 (1991).

[3] A. E. Miroshnichenko, S. Flach, and Y.S. Kivshar, Rev. Mod. Phys. 82, 2257 (2010).

[4] B. Luk'yanchuk, N. I. Zheludev, S. A. Maier, N. J. Halas, P. Nordlander, H. Giessen, and C. T. Chong, Nature Mater. 9, 707 (2010).

[5] J. P. Marangos, J. Mod. Opt. 45, 471 (1998).

[6] M. Fleischhauer, A. Imamoglu, and J. P. Marangos, Rev. Mod. Phys. 77, 633 (2005).

[7] S. Zhang, D. A. Genov, Y. Wang, M. Liu, and X. Zhang, Phys. Rev. Lett. 101, 047401 (2008).

[8] N. Liu, L. Langguth, T. Weiss, J. Kästel, M. Fleischhauer, T. Pfau, and H. Giessen, Nature Mater. 8, 758 (2009).

[9] V. Yannopapas, E. Paspalakis, and N. V. Vitanov, Phys. Rev. B 80, 035104 (2009).

[10] P. Tassin, L. Zhang, T. Koschny, E. N. Economou, and C.M. Soukoulis, Phys. Rev. Lett. 102, 053901 (2009).

[11] R. D. Kekatpure, E.S. Barnard, W. Cai, and M.L. Brongersma, Phys. Rev. Lett. 104, 243902 (2010).

[12] J. Zhang, W. Bai, L. Cai, Y. Xu, G. Song, and Q. Gan, Appl. Phys. Lett. 99, 181120 (2011).

[13] L. Hau, S. E. Harris, Z. Dutton, and C. H. Behroozi, Nature (London) 397, 594 (1999).

[14] C. Liu, Z. Dutton, C. H. Behroozi, and L. V. Hau, Nature (London) 409, 490 (2001).

[15] D. Bouwmeester, N. H. Dekker, F. E. v. Dorsselaer, C. A. Schrama, P. M. Visser, and J. P. Woerdman, Phys. Rev. A 51, 646 (1995).

[16] L. Novotny, Am. J. Phys. 78, 1199 (2010).

[17] P. Mühlschlegel, H.-J. Eisler, O. J. F. Martin, B. Hecht, and D. W. Pohl, Science 308, 1607 (2005).

[18] L. Novotny and N. van Hulst, Nature Photon. 5, 83 (2011).

[19] A. Christ, S. G. Tikhodeev, N. A. Gippius, J. Kuhl, and H. Giessen, Phys. Rev. Lett. 91, 183901 (2003).

[20] A. Alù and N. Engheta, Phys. Rev. Lett. 102, 233901 (2009).

[21] F. J. G. de Abajo, Physics 2, 47 (2009).

[22] S. Murai, M. A. Verschuuren, G. Lozano, G. Pirruccio, A. F. Koenderink, and J. G. Rivas, Opt. Express 2, 1111 (2012).

[23] A. Wokaun, H.-P. Lutz, A. P. King, U. P. Wild, and R. R. Ernst, J. Chem. Phys. 79, 509 (1983).

[24] P. Anger, P. Bharadwaj, and L. Novotny, Phys. Rev. Lett. 96, 113002 (2006).

[25] M. A. Verschuuren, Ph.D. thesis, Utrecht University, 2010.

[26] E. Palik, Handbook of Optical Constants of Solids (Academic Press, New York, 1985).

[27] A. Yariv and P. Yeh, Photonics: Optical Electronics in Modern Communications (Oxford University Press, Oxford, 2007), 6th ed.

[28] C. Alzar, M. Martinez, and P. Nussenzveig, Am. J. Phys. 70, 37 (2002).

[29] S. Mukherjee, H. Sobhani, J. B. Lassiter, R. Bardhan, P. Nordlander, and N. J. Halas, Nano Lett. 10, 2694 (2010).

[30] S. A. Maier, Plasmonics: Fundamentals and Applications (Springer, New York, 2007).

[31] C. Cohen-Tannoudji, J. Dupont-Roc, and G. Grynberg, Atom-Photon Interactions (Wiley-VCH, New York, 2004).

[32] J. Zuloaga and P. Nordlander, Nano Lett. 11, 1280 (2011). 